Article

\title{
Anomalous Electromagnetic Transport in Compact Stars
}

\author{
Efrain J. Ferrer * and Vivian de la Incera
}

Department of Engineering Science and Physics, CUNY-College of Staten Island and CUNY-Graduate Center, New York, NY 10314, USA; vivian.incera@csi.cuny.edu

* Correspondence: efrain.ferrer@csi.cuny.edu; Tel.: +1-718-982-2919

Received: 9 January 2018; Accepted: 13 February 2018; Published: 12 March 2018

\begin{abstract}
We study the anomalous electromagnetic transport properties of a quark-matter phase that can be realized in the presence of a magnetic field in the low-temperature/moderate-high-density region of the Quantum Chromodynamics (QCD) phase map. In this so-called Magnetic Dual Chiral Density Wave phase, an inhomogeneous condensate is dynamically induced producing a nontrivial topology, a consequence of the asymmetry of the lowest Landau level modes of the quasiparticles in this phase. The nontrivial topology manifests in the electromagnetic effective action via a chiral anomaly term $\theta F^{\mu v} \tilde{F}_{\mu v}$, with an axion field $\theta$ given by the phase of the Dual Chiral Density Wave condensate. The coupling of the axion with the electromagnetic field leads to several macroscopic effects that include, among others, an anomalous, nondissipative Hall current, an anomalous electric charge, magnetoelectricity, and the formation of a hybridized propagating mode known as an axion polariton. The possible existence of this phase in the inner core of neutron stars opens a window to search for signals of its anomalous transport properties.
\end{abstract}

Keywords: chiral symmetry; axion QED; quark-hole pairing; cold-dense QCD; magnetic DCDW

\section{Introduction}

Neutron stars, the remnants of supernova collapse, are very dense objects produced by the gravitational colapse of very massive stars (stars with masses between 10 and 30 solar masses). They can reach densities several times larger than the nuclear density of $4 \times 10^{17} \mathrm{~kg} / \mathrm{m}^{3}$. An interesting question that still remains unsettled is about the state of matter that supports such a dense medium. In this regard, very precise mass measurements for two compact objects, PSR J1614 - 2230 and PSR $\mathrm{J} 0348+0432$ with $M=1.97 \pm 0.04 M_{\odot}$ [1] and $M=2.01 \pm 0.04 M_{\odot}$ [2], respectively, where $M_{\odot}$ is the solar mass, have provided an important clue on the possible candidates for their interior composition: the phase of matter there should have an equation of state (EoS) rather stiff at high densities

The possibility of an interior composition based on a nucleon medium phase, formed mainly by neutrons, faces the difficulty of reproducing the required EoS stiffness. The reason is that, while under terrestrial conditions, hyperons are unstable and decay into nucleons through weak interactions, in neutron stars, the equilibrium conditions at core densities of order $2-3 \rho_{0}$ (with $\rho_{0} \simeq 0.16 \mathrm{fm}^{-3}$ the nuclear saturation density) can make the inverse process possible [3-12]. Thus, at large enough baryon chemical potential, the conversion of nucleons into hyperons becomes energetically favorable. This conversion releases the Fermi pressure exerted by the nucleons and makes the EoS soft enough to lead to a significant reduction of the star mass [4,13-20]. Different attempts to overcome this problem exist in the literature, as the inclusion of a repulsive hyperon-hyperon interaction through the exchange of vector mesons [21-25], or the inclusion of repulsive hyperonic three-body forces [26-31]. However, the possibility to reach the $2 M_{\odot}$ with a nucleon inner phase still remains an open question under discussion. 
On the other hand, in the highly dense cores of the compact objects, the neutron-rich matter can give rise to new degrees of freedom, by forming quark matter (see e.g., [32,33]). Even more, cold strange quark matter has been argued to be absolutely stable [34]. Thus, a phase transition can take place in the core favoring quark matter in the entire star interior, therefore giving rise to a strange star. Nevertheless, since the existence of $2 M_{\odot}$ stars $[35,36]$ was reported, there were also claims $[37,38]$ that quark matter has too soft an EoS to reach such a high-mass value. Nonetheless, it was later realized that to study quark matter in compact objects, one has to rely on effective models like the Nambu-Jona-Lasinio (NJL) models with parameters matched to nuclear data. The one-gluon exchange interaction of QCD contains a dominant attractive diquark channel that is incorporated as a four-fermion interaction term in NJL models. This attractive interaction gives rise to color superconductivity (CS) [39-42]. NJL models have predicted that the most favored phase of CS at asymptotically high densities is the three-flavor color-flavor-locked (CFL) phase with a significantly large gap. The existence of a large superconducting gap together with a repulsive vector interaction, which is always present in a dense medium [43], can help to make the EoS stiffer. Yet, it was also found more recently that gluons in the color superconducting medium can soften the EoS [44]. Then, it is not clear at present what will be the more significant factor among all these competing effects, since they depend on parameter values that cannot be fixed with total precision.

Moreover, even assuming that neutron stars can realize a quark phase, this does not take place at asymptotically large densities. Their location in the QCD phase map will better correspond to the low-temperature, intermediate-density region. This is a particularly challenging region with the possibility of spatially inhomogeneous phases. To visualize that, we should take into account that coming from the low density region, the energy separation between quarks and antiquarks grows with increasing density up to a point where to excite antiquarks all the way from the Dirac sea in order to pair them with the quarks at the Fermi surface is not energetically favorable anymore. In this case, instead of undergoing a transition to a chirally restored phase, the system prefers to pair quarks and holes with parallel momenta close to the Fermi surface, giving rise to inhomogeneous chiral condensates. Spatially inhomogeneous phases with quark-hole condensates have been found in the large-N limit of QCD [45-47], in quarkyonic matter [48-51], and in NJL models [52-57]. Hence, although most NJL models had predicted a first-order chiral transition with increasing density [58], it turned out that the transition is more likely to occur via some intermediate state(s) characterized by inhomogeneous chiral condensates.

Inhomogeneous phases become favored also in CS [59], when the intermediate density region is approached from the region of low temperatures and asymptotically high density values. As already pointed out, at asymptotic densities, the most favored CS phase is the CFL, a homogeneous phase in which all flavors pair with each other via the strong attractive quark-quark channel. This phase is based on BCS quark-pairing and relies on the assumption that the quarks that pair with equal and opposite momenta can each be arbitrarily close to their common Fermi surface. However, with decreasing density, the combined effect of the strange quark mass, neutrality constraint and beta equilibrium, tends to pull apart the Fermi momenta of different flavors, imposing an extra energy cost on the formation of Cooper pairs. Thus, we conclude that BCS-pairing dominates as long as the energy cost of forcing all species to have the same Fermi momentum is compensated by the pairing energy that is released by the formation of Cooper pairs.Then, with decreasing density, the CFL phase eventually becomes gapless and, most importantly, becomes unstable [60,61]. The instability, known as chromomagnetic instability, manifests itself in the form of imaginary Meissner masses for some of the gluons and indicates an instability towards spontaneous breaking of translational invariance [62-65]. In other words, it indicates the formation of a spatially inhomogeneous phase. Most inhomogeneous CS phases are based on the idea of Larkin and Ovchinnikov (LO) [66] and Fulde and Ferrell (FF) [67], originally applied to condensed matter. In the CS LOFF phases [68-70], quarks of different flavors pair even though they have different Fermi momenta, because they form Cooper pairs with nonzero momentum. CS inhomogeneous phases with gluon condensates that break rotational symmetry [71] 
have also been considered to remove the chromomagnetic instability. However, to the best of our knowledge, the question of which CS phase is the most favorable in the region of intermediate densities still remains unanswered.

In addition to their high densities, neutron stars typically have strong magnetic fields, which become extremely large in the case of magnetars, with inner values that have been estimated to range from $10^{18} \mathrm{G}$ for nuclear matter [72] to $10^{20} \mathrm{G}$ for quark matter [73]. The fact that strong magnetic fields populate the vast majority of the astrophysical compact objects and that they can significantly affect several properties of the star have served as motivation for many works focused on the study of the EoS of magnetized neutron stars, without considering [73-98], or considering the magnetic-field interaction with the particle anomalous-magnetic-moment [88,99-113]. An important characteristic is that the EoS in a uniform magnetic field becomes anisotropic, with different pressures along the field and transverse to it $[73,94,98]$. The transverse and longitudinal pressures can be found performing a quantum-statistical average of the energy-momentum tensor, as done in [73] using a path-integral approach based on the partition function of the grand canonical emsemble, or in [94] using the many-particle density matrix.

It has been found that as a consequence of the anisotropy in the EoS, the effects of the two pressures produce opposite contributions to the TOV equations. When the anisotropy becomes significant, the TOV approach is inadequate, since it is based on a medium with spherical symmetry, while the influence of a strong uniform magnetic field makes the geometry cylindrical. Lacking a suitable approach that is compatible with the symmetry of the problem, any conclusion about the effect of the magnetic field on the neutron star mass is in principle unreliable.

In summary, up to now it has been impossible to reliably determine if neutron stars (magnetars included) are formed by neutrons or quarks, or by a hybrid combination of them, and if quark matter is present, what its phase will be. That is why it is so important to look for new signals that can be attributed uniquely to a certain phase of the interior matter with the hope of using observations to pinpoint the inner composition of the star. Transport properties could be a way to reach this goal. This paper aims to advance such a strategy. We present the electromagnetic transport properties [114] of one of the quark phases: the Magnetic Dual Chiral Density Wave (MDCDW) phase, that can take place in the low-temperature/intermediate-density region of quark matter. An important attribute of this phase is its similarities with condensed matter topological materials like Weyl semimetals (WSM) $[115,116]$. This opens the possibility to take advantage of new understandings within these materials to infer potentially measurable effects in the MDCDW phase of quark matter, and then use that insight to design clever ways to probe the presence of this quark phase in neutron stars.

\section{The MCDCW Phase}

To study the electromagnetic properties of the MCDCW phase, we should start by modeling $\mathrm{QCD}+\mathrm{QED}$ with the help of the following Lagrangian density that combines electromagnetism with a two-flavor NJL model of strongly interacting quarks,

$$
\begin{aligned}
\mathcal{L}= & -\frac{1}{4} F_{\mu v} F^{\mu v}+\bar{\psi}\left[i \gamma^{\mu}\left(\partial_{\mu}+i Q A_{\mu}\right)+\gamma_{0} \mu\right] \psi \\
& +G\left[(\bar{\psi} \psi)^{2}+\left(\bar{\psi} i \tau \gamma_{5} \psi\right)^{2}\right]
\end{aligned}
$$

Here $Q=\operatorname{diag}\left(e_{u}, e_{d}\right)=\operatorname{diag}\left(\frac{2}{3} e,-\frac{1}{3} e\right), \psi^{T}=(u, d) ; \mu$ is the baryon chemical potential; and $\mathrm{G}$ is the four-fermion coupling. The electromagnetic potential $A^{\mu}$ is formed by the background $\bar{A}^{\mu}=(0,0, B x, 0)$, which corresponds to a constant and uniform magnetic field B pointing in the z-direction, with $x^{\mu}=(t, x, y, z)$, plus the fluctuation field $\tilde{A}$. In the presence of electromagnetic interactions the flavor symmetry $S U(2)_{L} \times S U(2)_{R}$ of the original NJL model is reduced to the subgroup $U(1)_{L} \times U(1)_{R}$. 
In the presence of a magnetic field, the formation of a dual chiral density wave (DCDW) condensate with magnitude $\Delta$ and modulation $q^{\mu}=(0,0,0, q)$ along the field direction $[117,118]$ is favored

$$
\langle\bar{\psi} \psi\rangle=\Delta \cos q_{\mu} x^{\mu}, \quad\left\langle\bar{\psi} i \tau_{3} \gamma_{5} \psi\right\rangle=\Delta \sin q_{\mu} x^{\mu},
$$

The mean-field Lagrangian of the MDCDW phase is then,

$$
\begin{aligned}
\mathcal{L}_{M F} & =\bar{\psi}\left[i \gamma^{\mu}\left(\partial_{\mu}+i Q A_{\mu}\right)+\gamma_{0} \mu\right] \psi-m \bar{\psi} e^{i \tau_{3} \gamma_{5} q_{\mu} x^{\mu}} \psi \\
& -\frac{m^{2}}{4 G}-\frac{1}{4} F_{\mu v} F^{\mu \nu},
\end{aligned}
$$

where $m=-2 G \Delta$.

To remove the spatial modulation of the mass, we use a local chiral transformation

$$
\psi \rightarrow e^{i \tau_{3} \gamma_{5} \theta} \psi, \quad \bar{\psi} \rightarrow \bar{\psi} e^{i \tau_{3} \gamma_{5} \theta}
$$

with $\theta(x)=q z / 2$.

After the chiral transformation (4), the mean-field Lagrangian density (3) becomes

$$
\begin{aligned}
\mathcal{L}_{M F} & =\bar{\psi}\left[i \gamma^{\mu}\left(\partial_{\mu}+i Q A_{\mu}+i \tau_{3} \gamma_{5} \partial_{\mu} \theta\right)+\gamma_{0} \mu-m\right] \psi \\
& -\frac{m^{2}}{4 G}-\frac{1}{4} F_{\mu \nu} F^{\mu \nu}
\end{aligned}
$$

The corresponding modified Dirac Hamiltonian of flavor $f$ is

$$
H_{f}=-i \gamma^{0} \gamma^{i}\left(\partial_{i}+i e_{f} A_{i}+i \frac{e_{f}}{\left|e_{f}\right|} \gamma_{5} \partial_{i} \theta\right)+\gamma^{0} m,
$$

Here $e_{f}$ is the flavor electric charge. The single-particle energy spectrum is given by the eigenvalues of $H_{f}$. It separates into two sets of energy modes [117], the LLL $(l=0)$ modes

$$
E^{0}=\epsilon \sqrt{m^{2}+k_{3}^{2}}+q / 2, \quad \epsilon= \pm
$$

and the higher Landau level $(l \neq 0)$ modes

$$
E^{l}=\epsilon \sqrt{\left(\xi \sqrt{m^{2}+k_{3}^{2}}+q / 2\right)^{2}+2\left|e_{f} B\right| l}, \quad \epsilon= \pm, \xi= \pm, l=1,2,3, \ldots
$$

In (8) $\xi= \pm$ indicates spin projection and $\epsilon= \pm$ particle/antiparticle energies. In contrast, only one spin projection ( + for positively charged and - for negatively charged quarks) contributes to the LLL spectrum. An important feature of this spectrum is that the LLL energies are not symmetric about the zero-energy level. As a consequence, the \pm sign in front of the square root should not be interpreted as particle/antiparticle in the LLL case.

A peculiarity of the local chiral transformation (4) is that it does not leave invariant the fermion measure in the path-integral. To take this into consideration, we need to calculate the contribution of the Jacobian $J(\theta(x))=\left(\operatorname{Det} U_{A}\right)^{-2}$ with $U_{A}=e^{i \tau_{3} \gamma_{5} \theta}$

$$
\mathcal{D} \bar{\psi}(x) \mathcal{D} \psi(x) \rightarrow\left(\operatorname{Det} U_{A}\right)^{-2} \mathcal{D} \bar{\psi}(x) \mathcal{D} \psi(x),
$$


to the effective action [114]. As a consequence, the fermion effective action entering in the partition function after the local chiral transformation is implemented reads

$$
\begin{aligned}
S_{e f f} & =\int d^{4} x\left\{\bar{\psi}\left[i \gamma^{\mu}\left(\partial_{\mu}+i Q A_{\mu}+i \tau_{3} \gamma_{5} \partial_{\mu} \theta\right)+\gamma_{0} \mu-m\right] \psi-\frac{m^{2}}{4 G}\right. \\
& \left.+\frac{\kappa}{4} \theta(x) F_{\mu \nu} \tilde{F}^{\mu v}\right\},
\end{aligned}
$$

on which $\frac{\kappa}{4}=\frac{3\left(e_{u}^{2}-e_{d}^{2}\right)}{8 \pi^{2}}=\frac{e^{2}}{8 \pi^{2}}=\frac{\alpha}{2 \pi}$ reflects the contribution of all the flavors and colors. The axion term $\frac{\kappa}{4} \theta(x) F_{\mu v} \tilde{F}^{\mu v}$ denotes the contribution of the Jacobian of the fermionic measure [114].

\section{Axion Electrodynamics in the MDCDW Phase}

To find the Maxwell equations of the MDCDW phase, we need to find the zero temperature electromagnetic effective action $\Gamma(A)$ corresponding to the effective classical action (10),

$$
\Gamma=-i \log Z,
$$

with $Z$ the partition function given by

$$
Z=e^{i \Gamma_{\text {matter }}}=\int \mathcal{D} \bar{\psi}(x) \mathcal{D} \psi(x) e^{i S_{\text {eff }}}
$$

with $S_{\text {eff }}$ given in (10).

After integrating in the fermion fields and carrying out the finite-temperature Matsubara sum to take the zero-temperature limit, we can expand $\Gamma$ in powers of the fluctuation field $\tilde{A}$ to obtain

$$
\begin{aligned}
\Gamma(A) & =-V \Omega+\int d^{4} x\left[-\frac{1}{4} F_{\mu v} F^{\mu v}+\frac{\kappa}{4} \theta(x) F_{\mu v} \tilde{F}^{\mu v}\right] \\
& +\sum_{i=1}^{\infty} \int d x_{1} \ldots d x_{i} \Pi^{\mu_{1}, \mu_{2}, \ldots \mu_{i}}\left(x_{1}, x_{2}, \ldots x_{i}\right) \tilde{A}_{\mu_{1}}\left(x_{1}\right) \ldots \tilde{A}_{\mu_{i}}\left(x_{i}\right),
\end{aligned}
$$

with $\mathrm{V}$ the four-volume, $\Omega$ the mean-field thermodynamic potential obtained for this phase in Ref. [117], and $\Pi^{\mu_{1}, \mu_{2}, \ldots \mu_{i}}$ the i-vertex tensors corresponding to the one-loop polarization operators with internal lines of fermion Green functions in the MDCDW phase and $i$ external lines of photons. In (13) we added the pure electromagnetic field contribution $-\frac{1}{4} F_{\mu v} F^{\mu v}$.

We are interested in the linear response of the MCDCW phase to a small electromagnetic probe $\tilde{A}$. Furthermore, for consistency of the approximation, we can neglect all the radiative corrections of order higher than $\alpha$, as $\alpha$ is the order of the axion term in (13). These two conditions imply that we shall cut the series in (13) at $i=1$, which can be shown to provide the medium corrections to the Maxwell equations that are linear in the electromagnetic field and of the desired order in $\alpha$.

Then, $\Gamma(A)$ becomes

$$
\begin{aligned}
\Gamma(A) & =-V \Omega+\int d^{4} x\left[-\frac{1}{4} F_{\mu v} F^{\mu \nu}-\kappa \int d^{4} x \epsilon^{\mu \alpha \nu \beta} A_{\alpha} \partial_{v} A_{\beta} \partial_{\mu} \theta\right] \\
& -\int d^{4} x \tilde{A}_{\mu}(x) J^{\mu}(x),
\end{aligned}
$$

where we integrated by parts the third term in the r.h.s. of $(13) . J^{\mu}(x)=\left(J^{0}, \mathbf{J}\right)$ represents the contribution of the ordinary (non-anomalous) electric four-current, determined by the one-loop tadpole diagrams. 
The Euler-Lagrange equations derived from the action (14) give rise to the modified Maxwell equations

$$
\begin{gathered}
\nabla \cdot \mathbf{E}=J^{0}+\frac{e^{2}}{4 \pi^{2}} q B \\
\nabla \times \mathbf{B}-\partial \mathbf{E} / \partial t=\mathbf{J}-\frac{e^{2}}{4 \pi^{2}} \mathbf{q} \times \mathbf{E}, \\
\nabla \cdot \mathbf{B}=0, \quad \nabla \times \mathbf{E}+\partial \mathbf{B} / \partial t=0,
\end{gathered}
$$

on which we already used that $\theta=\frac{q z}{2}$. These are the equations of axion electrodynamics for the MDCDW phase, which are a particular case of those proposed by Wilczek [119] many years ago for a general axion field $\theta$.

From Equations (15) and (16) we have that the axion term leads to an anomalous electric charge density,

$$
J_{\text {anom }}^{0}=\frac{e^{2}}{4 \pi^{2}} q B
$$

as well as to an anomalous Hall current density,

$$
\mathbf{J}_{\text {anom }}=-\frac{e^{2}}{4 \pi^{2}} \mathbf{q} \times \mathbf{E}
$$

The anomalous Hall current is perpendicular to both the magnetic and the electric field, since $\mathbf{q}$ is aligned with $\mathbf{B}$. Besides, $\mathbf{J}_{\text {anom }}$ is dissipationless and as such, it can significantly influence the transport properties of the system.

In (15) and (16), $J^{0}$ and $\mathbf{J}$ are the ordinary charge and current densities respectively, which are calculated from the tadpole diagrams of the theory. Of special interest is to check if the ordinary charge so obtained can annihilate the anomalous contribution $J_{\text {anom }}^{0}$. As discussed in [114], since $J_{\text {anom }}^{0}$ only gets contributions from the LLL, we should compare it with the corresponding tadpole result calculated in the LLL approximation. Then, calculating the tadpole diagrams we obtain

$$
J_{L L L}^{1,2,3}=0
$$

and

$$
\begin{aligned}
J_{L L L}^{0} & =\sum_{f} J_{L L L}^{0}\left(\operatorname{sgn}\left(e_{f}\right)\right) \\
& =\frac{e^{2} B}{2 \pi^{2}} \sqrt{(\mu-q / 2)^{2}-m^{2}}[\Theta(\mu-q / 2-m)-\Theta(q / 2-\mu-m)]
\end{aligned}
$$

Thus, there is no LLL current density, but only a charge density. Comparing Equations (18) and (21) we can see that they do not cancel out. Only if $m=0$ in (21), meaning setting the condensate amplitude to zero, the anomalous electric charge density will be cancelled out by the ordinary charge in Equation (15). In such a situation, the resulting LLL contribution to the net electric charge density reduces to $\frac{e^{2} B}{2 \pi^{2}} \mu$, a non-anomalous term which, as expected, is independent of $q$ since no physical quantity should depend on $q$ when there is no MDCDW condensate (i.e., when $m=0$ ).

Finally, since the Maxwell Equation (16) contains an anomalous Hall current, it is important to investigate if it can be cancelled out by an ordinary Hall current. The Hall conductivity can be easily found from the charge density in the case that it is linearly dependent on the magnetic field [114],

$$
\sigma_{x y}=\frac{\partial J^{0}}{\partial B}
$$


Applying this formula to the ordinary electric charge density (21), the corresponding LLL contribution to the Hall conductivity is

$$
\sigma_{x y}^{\text {ord }}=\frac{\partial J_{L L L}^{0}}{\partial B}=\frac{e^{2}}{2 \pi^{2}} \sqrt{(\mu-q / 2)^{2}-m^{2}}[\Theta(\mu-q / 2-m)-\Theta(q / 2-\mu-m)]
$$

which leads to the LLL ordinary Hall current $\mathbf{J}_{L L L}^{o r d}=\left(\sigma_{x y}^{\text {ord }} E_{y},-\sigma_{x y}^{\text {ord }} E_{x}, 0\right)$. Clearly, $\mathbf{J}_{L L L}^{\text {ord }}$ does not cancel out the anomalous current (19).

Likewise, the anomalous Hall conductivity can be found either from the anomalous charge (18),

$$
\sigma_{x y}^{a n o m}=\frac{\partial J_{\text {anom }}^{0}}{\partial B}=\frac{e^{2}}{4 \pi^{2}} q,
$$

or directly from the anomalous Hall current $\mathbf{J}_{\text {anom }}$ given in (19). As $J_{a n o m}^{0}$ is due to the LLL, so is $\sigma_{x y}^{a n o m}$, thereby underlining once again the LLL origin of $\mathbf{J}_{\text {anom }}$.

\section{Anomalous Transport in the MDCDW Phase}

The MDCDW phase exhibits some interesting electromagnetic features, such as for example, linear magnetoelectricity [114]. To see this, we should define the $\mathbf{D}$ and $\mathbf{H}$ fields as

$$
\mathbf{D}=\mathbf{E}-\kappa \theta \mathbf{B}, \quad \mathbf{H}=\mathbf{B}+\kappa \theta \mathbf{E}
$$

Then, rewriting the Maxwell Equations (15) and (16) in terms of these fields we obtain,

$$
\nabla \cdot \mathbf{D}=J^{0}, \quad \nabla \times \mathbf{H}-\frac{\partial \mathbf{D}}{\partial t}=\mathbf{J}
$$

Equations (25) imply that a magnetic field induces an electric polarization $\mathbf{P}=-\kappa \theta \mathbf{B}$ and an electric field induces a magnetization $\mathbf{M}=-\kappa \theta \mathbf{E}$. Both parameters, $\mathbf{P}$ and $\mathbf{M}$, depend on the theta angle, which evidences the anomalous character of this result. It is important to point out that the appearance of a linear magnetoelectricity in this medium is possible because, as seen from (25) and (26), the MDCDW ground state breaks parity and time-reversal symmetries. We call attention to the fact that the magnetoelectricity here is different from the one found in the magnetic-CFL phase of color superconductivity [120-122], where P was not broken and the effect was a consequence of an anisotropic electric susceptibility [123], so it was not linear.

In this formulation, it follows from (26) that the anomalous Hall current is given by a medium-induced, magnetic current density $\nabla \times \mathbf{M}$, due to the space-dependent anomalous magnetization coming from the axion term.

An interesting property of the anomalous Hall current is its dissipationless character. This is a consequence of the fact that this current (19) is perpendicular to $\mathbf{E}$ and to the modulation vector $\mathbf{q}$, which in turn is parallel to $\mathbf{B}$. We already proved that the anomalous Hall conductivity is given by

$$
\sigma_{x y}^{a n o m}=e^{2} q / 4 \pi^{2}
$$

Its anomalous character is reflected in the fact that it does not depend on the fermion mass $m$, which is consistent with the nondissipative character of the anomalous Hall current.

The same expression of the anomalous Hall conductivity has been found in WSM [115], where the role of the modulation parameter $q$ is played by the separation in momentum of the Weyl nodes. A similar Hall conductivity can appear also at the boundary between a topological and a normal insulator [124] when there is an electric field in the plane of the boundary. However, in the topological insulator case, the anomalous Hall conductivity is discrete because the axion field $\theta$ jumps from 0 to $\pi$ in the surface of the two insulators. Our results are also connected to optical lattices, as 3D topological insulators have been proposed to exist in 3D optical lattices [125]. 
It is worth pointing out the relevance of these results for neutron stars. The values of chemical potential and magnetic field needed for the realization of the MDCDW phase are all within the possible ranges of these parameters at the core of hybrid stars or in quark stars. As shown in [117], for baryon chemical potentials between 300 and $600 \mathrm{MeV}$, and magnetic field strengths $\sqrt{e B} \sim 150-500 \mathrm{MeV}$ $\left(\sim 10^{18}-10^{19} \mathrm{G}\right)$ the MDCDW is very robust, with $b$ and $m$ in the range between $300-500 \mathrm{MeV}$. The inhomogeneity parameter $b$ actually sets at arbitrarily small values of magnetic field and chemical potential because the anomalous term in the thermodynamic potential drives $b \neq 0$ from the beginning, but $b$ becomes of the order of the dynamical mass when the matter density becomes nonzero, which typically occurs when the chemical potential is comparable to the vacuum dynamical mass. Furthermore, the application to neutron stars requires introducing electrical neutrality (either locally or globally, as the neutrality does not need to be satisfied locally for compact hybrid stars [126]) and beta equilibrium conditions, as well as vector interactions to be able to accommodate the $2 M_{\odot}$ observations. None of these conditions however impede the realization of the MDCDW phase, as shown in detail in [93], where their main consequence was that each flavor acquires its own modulation and dynamical mass, and the MDCDW remains robust at fields of the order of $10^{18} \mathrm{G}$ and chemical potentials between 300 and $600 \mathrm{MeV}$. A significant result in [93] was the fact that the MDCDW phase is compatible with the $2 M_{\odot}$ observations. It is easy to see that the effect on the anomalous terms will simply be that each flavor still contributes to the anomalous electric charge and Hall current, only now each depends on the flavor's particular modulation parameter. The anomalous currents could serve to resolve the issue with the stability of the magnetic field strength in magnetars [127,128]. It will be worthy to understand if the magneto-transport properties of the MDCDW phase can significantly affect the thermal and electrical conductivity producing a tangible separation between the transport properties of compact stars formed by neutrons or by quarks in this inhomogeneous phase. These and other questions highlight the importance of exploring which observable signatures could be identified and then used as telltales of the presence of the MDCDW phase in the star core.

Another interesting effect can be connected to the fluctuations $\delta \theta$ of the axion field. If one goes beyond the mean-field approximation, there will be mass and kinetic terms of the axion field fluctuation. Besides, due to the background magnetic field, the axion fluctuation couples linearly to the electric field via the term $\kappa \delta \theta \mathbf{E} \cdot \mathbf{B}$, so the field equations of the axion fluctuation and the electromagnetic field will be mixed, giving rise to a quasiparticle mode known as the axion polariton mode [129]. The axion polariton mode is gapped with a gap proportional to the background magnetic field. This implies that electromagnetic waves of certain frequencies will be attenuated by the MDCDW matter, since in this medium they propagate as polaritons. The axion polariton could be useful to probe the presence of the MDCDW phase in different media.

\section{Conclusions}

In this paper we present the electromagnetic anomalous transport properties of the so called MDCDW phase of quark matter at low temperatures and moderate densities in the presence of a magnetic field. This is a phase that in principle can be realized in neutron hybrid stars with a quark core or in strange stars.

The system under study has a non-trivial topology, which is due to the combined effect of a ground state having an inhomogeneous particle-hole condensate and the dimensional reduction affecting the quasiparticles occupying the LLL. As a consequence, the system exhibits an anomalous charge that depends on the applied magnetic field and the modulation of the particle-hole condensate. The topological nature of the electric charge can be traced back to the spectral asymmetry of the LLL modes. The spectral asymmetry is also responsible for an anomalous non-dissipative Hall current that depends on the modulation parameter.

We should mention that the reported results can also have importance for Heavy Ion Collision (HIC) physics. Future HIC experiments, that will take place at lower temperatures and higher densities, will certainly generate strong magnetic and electric fields in their off-central collisions and will open 
a much more sensitive window to look into a very challenging region of QCD [130]. For example, the second phase of the RHIC energy scan (BES-II) [131], the planned experiments at the Facility for Antiproton and Ion Research (FAIR) [132] at the GSI site in Germany, and the Nuclotron-based Ion Collider Facility (NICA) $[133,134]$ at JINR laboratory in Dubna, Russia, are all designed to run at unprecedented interaction rates to provide high-precision measures of observables in the high baryon density region. That is why it is so timely and relevant to carry out detailed theoretical investigations of all potential observables of the MDCDW phase. Therefore, we hope that our findings will serve to stimulate quantitative studies to identify signatures of the anomalous effects here discussed in the future HIC experiments.

In addition, we should notice that the anomalous effects of the MDCDW phase share many properties with similar phenomena in condensed matter systems with non-trivial topologies as topological insulators [124], where $\theta$ depends on the band structure of the insulator; Dirac semimetals [135-138], a 3D bulk analogue of graphene with non-trivial topological structures; and WSM [115], where the derivative of the angle $\theta$ is related to the momentum separation between the Weyl nodes. Countertop experiments with these materials can therefore help us to gain useful insight into the physics governing the challenging region of strongly coupled QCD, thereby inspiring new strategies to probe the presence of the MDCDW phase in neutron stars and HIC.

Acknowledgments: This work was supported in part by NSF grant PHY-1714183, and PSC-CUNY Award 60650-00 48 .

Author Contributions: These authors contributed equally to this work.

\section{References}

1. Demorest, P.; Pennucci, T.; Ransom, S.M.; Roberts, M.S.E.; Hessels, J.W.T. Shapiro delay measurement of a two solar mass neutron star. Nature 2010, 467, 1081-1083.

2. Antoniadis, J.; Paulo, C.C.; Freire, P.C.C.; Wex, N.; Tauris, T.M.; Lynch, R.S.; van Kerkwijk, M.H.; Kramer, M.; Bassa, C.; Dhillon, V.S.; et al. A massive pulsar in a compact relativistic binary. Science 2013, 340, 1233232.

3. Ambartsumyan, V.A.; Saakyan, G.S. The degenerate superdense gas of elementary particles. Sov. Astron. 1960, 4, 187-201.

4. Glendenning, N.K. The hyperon composition of neutron stars. Phys. Lett. B 1982, 114, 392-396.

5. Glendenning, N.K. Neutron stars are giant hypernuclei? Astrophys. J. 1985, 293, 470-493.

6. Weber, F.; Weigel, M.K. Baryon composition and macroscopic properties of neutron stars. Nucl. Phys. A 1989, 505, 779-822.

7. Knorren, R.; Prakash, M.; Ellis, P.J. Strangeness in hadronic stellar matter. Phys. Rev. C 1995, 52, 3470-3482.

8. Schulze, H.-J.; Sagawa, H.; Wu, C.-X.; Zhao, E.-G. Hypernuclei in the deformed Skyrme-Hartree-Fock approach. Phys. Rev. C 2007, 76, 034312.

9. Zhou, X.-R.; Polls, A.; Schulze, H.-J.; Vidaña, I. Lambda hyperons and the neutron drip line. Phys. Rev. C 2008, 78, 054306.

10. Sammarruca, F. Effects of Lambda hyperons on the nuclear equation of state in a Dirac-Brueckner-Hartree-Fock model. Phys. Rev. C 2009, 79, 034301.

11. Lonardoni, D.; Pederiva, F.; Gandolfi, S. Accurate determination of the interaction between hyperons and nucleons from auxiliary field diffusion Monte Carlo calculations. Phys. Rev. C 2014, 89, 014314.

12. Katayama, T.; Saito, K. Hyperons in neutron stars. Phys. Lett. B 2015, 747, $43-47$.

13. Glendenning, N.K. Hyperons in neutron stars. Z. Phys. A 1987, 326, 57-64.

14. Schulze, H.-J.; Baldo, M.; Lombardo, U.; Cugnon, J.; Lejeune, A. Hypernuclear matter in the Bruckner-Hartree-Fock approximation. Phys. Lett. B 1995, 355, 21-26.

15. Schulze, H.-J.; Baldo, M.; Lombardo, U.; Cugnon, J.; Lejeune, A. Hyperonic nuclear matter in Bruckner theory. Phys. Rev. C 1998, 57, 704.

16. Baldo, M.; Burgio, G.F.; Schulze, H.-J. Hyperon stars in the Brueckner-Bethe-Goldstone theory. Phys. Rev. C 2000, 61, 055801.

17. Vidaña, I.; Polls, A.; Ramos, A.; Engvik, L.; Hjorth-Jensen, M. Hyperon-hyperon interactions and properties of neutron star matter. Phys. Rev. C 2000, 62, 035801. 
18. Schulze, H.-J.; Polls, A.; Ramos, A.; Vidaña, I. Maximum mass of neutron stars. Phys. Rev. C 2006, $73,058801$.

19. Djapo, H.; Schaefer, B.-J.; Wambach, J. Appearance of hyperons in neutron stars. Phys. Rev. C 2010, 81, 035803.

20. Schulze, H.-J.; Rijken, T. Maximum mass of hyperon stars with the Nijmegen ESC-08 model. Phys. Rev. C 2011, 84, 035801.

21. Bednarek, I.; Haensel, P.; Zdunik, L.; Bejger, M.; Mánka, R. Hyperons in neutron-star cores and two-solar-mass pulsar. Astron. Astrophys. 2012, 157, 543.

22. Weissenborn, S.; Chatterjee, D.; Schaffner-Bielich, J. Hyperons and massive neutron stars: vector repulsion and SU(3) symmetry. Phys. Rev. C 2012, 85, 065802.

23. Weissenborn, S.; Chatterjee, D.; Schaffner-Bielich, J. Hyperons and massive neutron stars: vector repulsion and SU(3) symmetry (Erratum). Phys. Rev. C 2014, 90, 019904.

24. Oertel, M.; Providência, C.; Gulminelli, F.; Raduta, A.R. Hyperons in neutron star matter within relativistic mean-field models. J. Phys. G 2015, 42, 075202.

25. Maslov, K.A.; Kolomeitsev, E.E.; Voskresensky, D.N. Solution of the hyperon puzzle within a relativistic mean-field model Phys. Lett. B 2015, 748, 369-375.

26. Takatsuka, T.; Nishizaki, S.; Yamamoto, Y. Necessity of extra repulsion in hypernuclear systems: Suggestion from neutron stars. Eur. Phys. J. A 2002, 13, 213.

27. Takatsuka, T.; Nishizaki, S.; Tamagaki, R. Three-Body force as an extra repulsion suggested from hyperon-mixed neutron stars. Prog. Theor. Phys. Suppl. 2002, 174, 80-83.

28. Vidaña, I.; Logoteta, D.; Providência, C.; Polls, A.; Bombaci, I. Estimation of the effect of hyperonic three-body forces on the maximum mass of neutron stars. Eur. Phys. Lett. 2011, 94, 11002.

29. Yamamoto, Y.; Furumoto, T.; Yasutake, B.; Rijken, T.A. Multi-pomeron repulsion and the neutron-star mass. Phys. Rev. C 2013, 88, 022801.

30. Yamamoto, Y.; Furumoto, T.; Yasutake, B.; Rijken, T.A. Hyperon mixing and universal many-body repulsion in neutron stars. Phys. Rev. C 2014, 90, 045805.

31. Lonardoni, D.; Lovato, A.; Gandolfi, S.; Pederiva, F. Hyperon puzzle: Hints from Quantum Monte Carlo calculations. Phys. Rev. Lett. 2015, 114, 092301.

32. Weber, F. Strange quark matter and compact stars. Prog. Part Nucl. Phys. 2005, 54, 193-288.

33. Lattimer, J.M. Neutron star equation of state. New Astron. Rev. 2010, 54, 101.

34. Witten, E. Cosmic separation of phases. Phys. Rev. D 1984, 30, 272.

35. Nice, D.J.; Splaver E.M.; Stairs, I.H.; Loehmer, O.; Jessner, A.; Kramer, M.; Cordes, J.M. A 2.1 solar mass pulsar measured by relativistic orbital decay. Astrophys. J. 2005, 634, 1242-1249.

36. Barret, D.; Olive, J.F.; Miller, M.C. An abrupt drop in the coherence of the lower kilohertz QPO in 4U 1636-536. Mon. Not. Roy. Astron. Soc. 2005, 361, 855-860.

37. Trümper, J.E.; Burwitz, V.; Haberl, F.; Zavlin, V.E. The puzzles of RX J1856.5-3754: Neutron star or quark star? Nucl. Phys. Proc. Suppl. 2004, 132, 560-565.

38. Özel, F. Soft equations of state for neutron-star matter ruled out by EXO 0748-676. Nature 2006, 441, 1115-1117.

39. Alford, M.G.; Schmitt, A.; Rajagopal, K.; Schäfer, T. Color superconductivity in dense quark matter. Rev. Mod. Phys. 2008, 80, 1455-1515.

40. Huang, M. QCD phase diagram at high temperature and density. arXiv 2010, arXiv:1001.3216.

41. Schmitt, A. Dense Matter in Compact Stars, Lecture Notes in Physics v. 811; Springer: Berlin/Heidelberg, Germany, 2010.

42. Fukushima, K.; Hatsuda, T. The phase diagram of dense QCD. Rept. Prog. Phys. 2011, 74, 014001.

43. Kitazawa, M.; Koide, T.; Kunihiro, T.; Nemoto, Y. Chiral and color superconducting phase transitions with vector interaction in a simple model. Prog. Theor. Phys. 2002, 108, 929-951.

44. Ferrer, E.J.; de la Incera, V.; Paulucci, L. Gluon effects on the equation of state of color superconducting strange stars. Phys. Rev. D 2015, 92, 043010.

45. Deryagin, D.V.; Grigoriev, D.Y.; Rubakov, V.A. Standing wave ground state in high density, zero temperature QCD at large N(c). Int. J. Mod. Phys. A 1992, 7, 659-681.

46. Shuster, E.; Son, D.T. On finite density QCD at large N(c). Nucl. Phys. B 2000,573, 434-446.

47. Park, B.-Y.; Rho, M.; Wirzba, A.; Zahed, I. Dense QCD: Overhauser or BCS pairing? Phys. Rev. D 2000, 62, 034015. 
48. Kojo, T.; Hidaka, Y.; McLerran, L.; Pisarski, R.D. Quarkyonic chiral spirals. Nucl. Phys. A 2010, 843, 37-58.

49. Kojo, T.; Hidaka, Y.; Fukushima, K.; McLerran, L.D.; Pisarski, R.D. Interweaving chiral spirals. Nucl. Phys. A 2012, 875, 94-138.

50. Kojo, T.; Pisarski, R.D.; Tsvelik, A.M. Covering the Fermi surface with patches of quarkyonic chiral spirals. Phys. Rev. D 2010, 82, 074015.

51. Kojo, T. A (1+1) dimensional example of quarkyonic matter. Nucl. Phys. A 2012, 877, 70-94.

52. Nickel, D. How many phases meet at the chiral critical point? Phys. Rev. Lett. 2009, 103, 072301.

53. Nickel, D. Inhomogeneous phases in the Nambu-Jona-Lasino and quark-meson model. Phys. Rev. D 2009, 80, 074025.

54. Rapp, R.; Shuryak, E.; Zahed, I. A Chiral crystal in cold QCD matter at intermediate densities? Phys. Rev. D 2001, 63, 034008.

55. Gubina, N.V.; Klimenko, K.G.; Kurbanov, S.G.; Zhukovsky, V.C. Inhomogeneous charged pion condensation phenomenon in the $\mathrm{NJL}_{2}$ model with quark number and isospin chemical potentials. Phys. Rev. D 2012, 86, 085011.

56. Carignano, S.; Nickel, D.; Buballa, M. Influence of vector interaction and Polyakov loop dynamics on inhomogeneous chiral symmetry breaking phases. Phys. Rev. D 2010, 82, 054009.

57. Abuki, H.; Ishibashi, D.; Suzuki, K. Crystalline chiral condensates off the tricritical point in a generalized Ginzburg-Landau approach. Phys. Rev. D 2012, 85, 074002.

58. Klevansky, S. The Nambu-Jona-Lasinio model of quantum chromodynamics. Rev. Mod. Phys. 1992, 64, 649-708.

59. Anglani, R.; Casalbuoni, R.; Ciminale, M.; Ippolito, N.; Gatto, R.; Mannarelli, M.; Ruggieri, M. Crystalline color superconductors. Rev. Mod. Phys. 2014, 86, 509-561.

60. Casalbuoni, R.; Gatto, R.; Mannarelli, M.; Nardulli, G.; Ruggieri, M. Meissner masses in the gCFL phase of QCD. Phys. Lett. B 2005, 605, 362-368.

61. Fukushima, K. Analytical and numerical evaluation of the Debye and Meissner masses in dense neutral three-flavor quark matter. Phys. Rev. D 2005, 72, 074002.

62. Reddy, S.; Rupak, G. Phase structure of 2-flavor quark matter: Heterogeneous superconductors. Phys. Rev. C 2005, 71, 025201.

63. Fukushima, K. Characterizing the Larkin-Ovchinnikov-Fulde-Ferrel phase induced by the chromomagnetic instability. Phys. Rev. D 2006, 73, 094016.

64. Hashimoto, M. Manifestation of instabilities in Nambu-Jona-Lasinio type models. Phys. Lett. B 2006, 642, 93-99.

65. Huang, M. Spontaneous current generation in the 2SC phase. Phys. Rev. D 2006, 73, 045007.

66. Larkin, A.I.; Ovchinnikov, Y.N. Nonuniform state of superconductors. Sov. Phys. JETP 1965, 20, 762-770.

67. Fulde, P.; Ferrell, R.A. Superconductivity in a strong spin-exchange field. Phys. Rev. 1964, 135, A550-A563.

68. Alford, M.G.; Bowers, J.A.; Rajagopal, K. Crystalline color superconductivity. Phys. Rev. D 2001, 63, 074016.

69. Bowers, J.A.; Rajagopal, K. The crystallography of color superconductivity. Phys. Rev. D 2001, 66, 065002.

70. Casalbuoni, R.; Nardulli, G. Inhomogeneous superconductivity in condensed matter and QCD. Rev. Mod. Phys. 2004, 76, 263-320.

71. Ferrer, E.J.; de la Incera, V. Chromomagnetic instability and induced magnetic field in neutral two-flavor color superconductivity. Phys. Rev. D 2007, 76, 114012.

72. Dong, L; Shapiro, S. L. Cold equation of state in a strong magnetic field - Effects of inverse beta-decay. ApJ 1991, 383, 745-751.

73. Ferrer, E.J.; de la Incera, V.; Keith, J.P.; Portillo, I.; Springsteen, P.L. Equation of state of a dense and magnetized fermion system. Phys. Rev. C 2010, 82, 065802.

74. Fushiki, I.; Gudmundsson, E.H.; Pethick, C.J. Surface structure of neutron stars with high magnetic fields. Astrophys. J. 1989, 342, 958-975.

75. Abrahams, A.M.; Shapiro, S.L. Equation of state in a strong magnetic field-Finite temperature and gradient corrections. Astrophys. J. 1991, 374, 652-667.

76. Fushiki, I.; Gudmundsson, E H.; Yngvason, J.; Pethick, C.J. Matter in a magnetic field in the Thomas-Fermi and related theories. Ann. Phys. 1992, 216, $29-72$.

77. Chakrabarty, S.; Bandyopadhyay, D.; Pal, S. Dense nuclear matter in a strong magnetic field. Phys. Rev. Lett. 1997, 78, 2898-2901. 
78. Bandyopadhyay, D.; Chakrabarty, S.; Dey, P. Rapid cooling of magnetized neutron stars. Phys. Rev. D 1998, $58,121301$.

79. Broderick, A.; Prakash, M.; Lattimer, J.M. The Equation of state of neutron star matter in strong magnetic fields. Astrophys. J. 2000, 537, 351-367.

80. Cardall, C.Y.; Prakash, M.; Lattimer, J.M. Effects of strong magnetic fields on neutron star structure. Astrophys. J. 2001, 554, 322-339.

81. Suh, I.-S.; Mathews, G.J. Cold ideal equation of state for strongly magnetized neutron star matter: Effects on muon production and pion condensation. Astrophys. J. 2001, 546, 1126-1136.

82. Perez-Martinez, A.; Perez-Rojas, H.; Mosquera-Cuesta, H.J. Magnetic collapse of a neutron gas: Can magnetars indeed be formed? Eur. Phys. J. C 2003, 29, 111-123.

83. Wei, F.X.; Mao, G.J.; Ko, C.M.; Kisslinger, L.S.; Stoecker, H.; Greiner, W. Effect of isovector-scalar meson on neutron star matter in strong magnetic fields. J. Phys. G 2006, 32, 47-61.

84. Hardings, A.K.; Lai, D. Physics of strongly magnetized neutron stars. Rep. Prog. Phys. 2006, 69, 2631.

85. Chen, W.; Zhang, P.-Q.; Liu, L.-G. The influence of the magnetic field on the properties of neutron star matter. Mod. Phys. Lett. A 2007, 22, 623-630.

86. Rabhi, A.; Providencia, C.; da Providencia, J. Stellar matter with a strong magnetic field within density-dependent relativistic models. J. Phys. G 2008, 35, 125201.

87. Gonzalez-Felipe, R.; Perez-Martinez, A.; Perez-Rojas, H.; Orsaria, M. Magnetized strange quark matter and magnetized strange quark stars. Phys. Rev. C 2008, 77, 015807.

88. Yue, P.; Yang, F.; Shen, H. Properties of hyperonic matter in strong magnetic fields. Phys. Rev. C 2009, 79, 025803.

89. Menezes, D.P.; Benghi Pinto, M.; Avancini, S. S.; Perez Martinez, A.; Providencia, C. Quark matter under strong magnetic fields in the Nambu-Jona-Lasinio Model. Phys. Rev. C 2009, 79, 035807.

90. Menezes, D.P.; Benghi Pinto, M.; Avancini, S. S.; Providencia, C. Quark matter under strong magnetic fields in the SU(3) Nambu-Jona-Lasinio model. Phys. Rev. C 2009, 80, 065805.

91. Paulucci, L.; Ferrer, E.J.; de la Incera, V.; Horvath, J.E. Equation of state for the MCFL phase and its implications for compact star models. Phys. Rev. D 2011, 83, 043009.

92. Fayazbakhsh, S.; Sadooghi, N. Anomalous magnetic moment of hot quarks, inverse magnetic catalysis, and reentrance of the chiral symmetry broken phase. Phys. Rev. D 2014, 90, 105030.

93. Carignano, S.; Ferrer, E.J.; de la Incera, V.; Paulucci, L. Crystalline chiral condensates as a component of compact stars. Phys. Rev. D 2015, 92, 105018.

94. Canuto, V.; Chiu, H.Y. Quantum theory of an electron gas in intense magnetic fields. Phys. Rev. 1968, 173, 1210-1219.

95. Canuto, V.; Chiu, H.Y. Thermodynamic properties of a magnetized fermi gas. Phys. Rev. 1968, 173, 1220.

96. Canuto, V.; Chiu, H.Y. Magnetic moment of a magnetized fermi gas. Phys. Rev. 1968, 173, 1229-1235.

97. Chiu, H.Y.; Canuto, V.; Fassio-Canuto, L. Quantum Theory of an Electron Gas with Anomalous Magnetic Moments in Intense Magnetic Fields. Phys. Rev. 1968, 176, 1438-1442.

98. Chaichian, M.; Masood, S.S.; Montonen, C.; Perez Martinez, A.; Perez Rojas, H. Quantum magnetic and gravitational collapse. Phys. Rev. Lett. 2000, 84, 5261-5264.

99. Chakrabarty, S. Quark matter in strong magnetic field. Phys. Rev. D 1996, 54, 1306-1316.

100. Broderick, A.; Prakash, M.; Lattimer, J.M. Effects of strong magnetic fields in strange baryonic matter. Phys. Lett. B 2002, 531, 167-174.

101. Khalilov, V.R. Macroscopic effects in cold magnetized nucleons and electrons with anomalous magnetic moments. Phys. Rev. D 2002, 65, 056001.

102. Mao, G.; Kondratyev, V. N.; A. Iwamoto, A.; Li, Z.; Wu, X.; Greiner, W. Neutron star composition in strong magnetic fields. Chin. Phys. Lett. 2003, 20, 1238-1241.

103. Mao, G.; Iwamoto, A.; Li, Z. Study of the neutron star structure in strong magnetic fields including the anomalous magnetic moments. Chin. J. Astron. Astrophys. 2003, 3, 359-374.

104. Perez Martinez, A.; Perez Rojas, H.; Mosquera Cuesta, H. J.; Boligan, M.; Orsaria, M. G. Quark stars and quantum-magnetically induced collapse. Int. J. Mod. Phys. D 2005, 14, 1959.

105. Felipe, R.G.; Martinez, A.P.; Rojas, H.P.; Orsaria, M. Magnetized strange quark matter and magnetized strange quark stars. Phys. Rev. C 2008, 77, 015807. 
106. Perez-Garcia, M.A.; Navarro, J.; Polls, A. Neutron Fermi Liquids under the presence of a strong magnetic field with effective nuclear forces. Phys. Rev. C 2009, 80, 025802.

107. Rabhi, A.; Pais, H.; Panda, P.K.; Providencia, C.J. Quark-hadron phase transition in a neutron star under strong magnetic fields. Phys. G 2009, 36, 115204.

108. Dexheimer, V.; Negreiros, R.; Schramm, S. Hybrid Stars in a strong magnetic field. Eur. Phys. J. A 2012, 48, 189.

109. Strickland, M.; Dexheimer, V.; Menezes, D.P. Bulk properties of a Fermi gas in a magnetic field. Phys. Rev. D 2012, 86, 125032.

110. Dong, J.; Lombardo, U.; Zuo, W.; Zhang, H. Dense nuclear matter and symmetry energy in strong magnetic fields. Nucl. Phys. A 2013, 898, 32-42.

111. Casali, R.H.; Castro, L.B.; Menezes, D.P. Hadronic and hybrid stars subject to density dependent magnetic fields. Phys. Rev. C 2014, 89, 015805.

112. Manreza Paret, D.; Perez Martinez, A.; Ferrer, E.J.; de la Incera, V. Effects of AMM on the EoS of magnetized dense systems. Astron. Nachr. 2014, 335, 685-690.

113. Ferrer, E.J.; de la Incera, V.; Manreza Peret, D.; Perez Martinez, A.; Sanchez, A. Insignificance of the anomalous magnetic moment of charged fermions for the equation of state of a magnetized and dense medium. Phys. Rev. D 2015, 91, 085041.

114. Ferrer, E.J.; de la Incera, V. Dissipationless Hall current in dense quark matter in a magnetic field. Phys. Lett. B 2017, 69, 208-212.

115. Burkov, A.A.; Balents, I. Weyl semimetal in a topological insulator multilayer. Phys. Rev. Lett. 2011, 107, 127205.

116. Zyuzin, A.A.; Burkov, A.A. Topological response in Weyl semimetals and the chiral anomaly. Phys. Rev. B 2012, 86, 115133.

117. Frolov, I.E.; Zhukovsky, V.C.; Klimenko, K.G. Chiral density waves in quark matter within the Nambu-Jona-Lasinio model in an external magnetic field. Phys. Rev. D 2010, 82, 076002.

118. Tatsumi, T.; Nishiyama, K.; Karasawa, S.Novel Lifshitz point for chiral transition in the magnetic field. Phys. Lett. B 2015, 743, 66-70.

119. Wilczek, F. Two Applications of Axion Electrodynamics. Phys. Rev. Lett. 1987, 58, 1799-1802.

120. Ferrer, E.J.; de la Incera, V.; Manuel, C. Magnetic color flavor locking phase in high density QCD. Phys. Rev. Lett. 2005, 152002.

121. Ferrer, E.J.; de la Incera, V.; Manuel, C. Color-superconducting gap in the presence of a magnetic field. Nucl. Phys. B 2006, 747, 88-112.

122. Ferrer, E.J.; de la Incera, V.; Manuel, C. Colour superconductivity in a strong magnetic field. J. Phys. A 2006, 39, 6349-6355.

123. Feng, B.; Ferrer, E.J.; de la Incera, V. Magnetoelectric effect in strongly magnetized color superconductivity. Phys. Lett. B 2011, 706, 232-238.

124. Qi, X.-L.; Hughes, T.L.; Zhang, S.-C. Topological field theory of time-reversal invariant insulators. Phys. Rev. B 2008, 78, 195424.

125. Bermudez, A.; Mazza, L.; Rizzi, M.; Goldman, N.; Lewenstein, M.; Martin-Delgado, M.A. Wilson fermions and axion electrodynamics in optical lattices. Phys. Rev. Lett. 2010, 105, 190404.

126. Glendenning, N.K. Compact Stars, Nuclear Physics, Particle Physics, General Relativity; Springer: New York, NY, USA, 2000.

127. Harding, A.K.; Lai, D. Physics of strongly magnetized neutron stars. Rept. Prog. Phys. 2006, 69, 2631-2708.

128. Spruit, H.C. Origin of neutron star magnetic fields. AIP Conf. Proc. 2008, 983, 391-398.

129. Li, R.; Wang, J.-L.; Qi, X.-C.; Zhang, S. Dynamical axion field in topological magnetic insulators. Nat. Phys. 2010, 6, 284-288.

130. Ferrer, E.J.; de la Incera, V. Exploring dense and cold QCD in magnetic fields. Eur. Phys. J. A 2016, 52, 266.

131. Odyniec, G. The RHIC beam energy scan program in STAR and what's next ... J. Phys. Conf. Ser. 2013, 455, 012037.

132. Ablyazimov, T.; Abuhoza, A.; Adak, P.P.; Adamczyk, M.; Agarwal, K.; Aggarwal, M.M.; Ahammed, Z.; Ahmad, F.; Ahmad, N.; Ahmad, S.; et al. [CBM Collaboration] Challenges in QCD matter physics -The scientific program of the Compressed Baryonic Matter experiment at FAIR. Eur. Phys. J. A 2017, 53, 60. 
133. Deng, W.-T.; Huang, X.-G. Event-by-event generation of electromagnetic fields in heavy-ion collisions. Phys. Rev. C 2012, 85, 044907.

134. Toneev, V.; Rogachevsky, O.; Voronyuk, V. Evidence for creation of strong electromagnetic fields in relativistic heavy-ion collisions. Eur. Phys. J. A 2016, 52, 264.

135. Young, S.M.; Zaheer, S.; Teo, J.C.Y.; Kane, C.L.; Mele, E.J.; Rappe, A.M. Dirac semimetal in three dimensions. Phys. Rev. Lett. 2012, 108, 140405.

136. Borisenko, S.; Gibson, Q.; Evtushinsky, D.; Zabolotnyy, V.; Buchner, B.; Cava, R.J. Experimental realization of a three-dimensional Dirac semimetal. Phys. Rev. Lett. 2014, 113, 027603.

137. Neupane, M.; Xu, S.Y.; Sankar, R.; Alidoust, N.; Bian, G.; Liu, C.; Belopolski, I.; Chang, T.R.; Jeng, H. T.; Lin, H.; Bansil, A; et al. Observation of a three-dimensional topological Dirac semimetal phase in high-mobility $\mathrm{Cd}_{3} A s_{2}$. Nat. Commun. 2014, 5, 3786.

138. Liu, Z.K.; Jiang, J.; Zhou, B.; Wang, Z.J.; Zhang, Y.; Weng, H.M.; Prabhakaran, D.; Mo, S.-K.; Peng, H.; Dudin, P.; et al. A stable three-dimensional topological Dirac semimetal $C d_{3} A s_{2}$. Nat. Mat. 2014, 13, 677-681.

(C) 2018 by the authors. Licensee MDPI, Basel, Switzerland. This article is an open access article distributed under the terms and conditions of the Creative Commons Attribution (CC BY) license (http:/ / creativecommons.org/licenses/by/4.0/). 